\title{
Robustness and uncertainties in global multivariate wind- wave climate projections
}

Joao Morim ${ }^{*}, 1,2$
, Mark Hemer

${ }^{1}$ Griffith University, School of Built Environment and Engineering, Southport, Queensland, Australia.

${ }^{2}$ Commonwealth Scientific and Industrial Research Organisation (CSIRO) Oceans and Atmosphere,

Hobart, Tasmania, Australia.

${ }^{3}$ Environment and Climate Change Canada, Climate Research Division, Toronto, Ontario, Canada.

${ }^{6}$ Department of Water Science and Engineering, IHE-Delft, Delft, The Netherlands.

${ }^{7}$ Faculty of Sciences, University of Lisbon, Lisbon, Portugal.

${ }^{8}$ National Oceanographic Centre, Liverpool, United Kingdom.

${ }^{9}$ Environmental Hydraulics Institute IH Cantabria, Universidad de Cantabria, Santander, Spain.

${ }^{10}$ US Geological Survey (USGS), Pacific Coastal and Marine Science Center, Santa Cruz, California, USA.

${ }^{11}$ Joint Research Centre, European Commissions Science Service, Savona, Italy.

${ }^{12}$ Disaster Prevention Research Institute, Kyoto University, Kyoto, Japan.

${ }^{13}$ Climate and Ecosystems Science Division, Lawrence Berkeley National Laboratory (LBNL),

Berkeley, California, USA.

${ }^{14}$ Norwegian Meteorological Institute, Bergen, Norway.

${ }^{15}$ Geophysical Institute, University of Bergen, Bergen, Norway.

${ }^{16}$ Helmholtz-Zentrum Geesthacht Centre for Materials and Coastal Research, Geesthacht, Germany.

${ }^{17}$ Institute of Oceanography, Center for Earth System Research and Sustainability (CEN), Universität

Hamburg, Hamburg, Germany.

${ }^{18}$ Computational Research Division, Lawrence Berkeley National Laboratory (LBNL), Berkeley,

California, USA.

${ }^{19}$ The Hakubi Center for Advanced Research and Disaster Prevention Research Institute, Kyoto

University, Kyoto, Japan.

${ }^{20}$ Department of Ocean and Resources Engineering, University of Hawai'i a at Mānoa, Honolulu, Hawaii, USA.

${ }^{21}$ Department of Infrastructure Engineering, University of Melbourne, Parkville, Victoria, Australia.

*Corresponding author address:

Eng. Joao Morim

Griffith University, School of Built Environment and Engineering (G39)

Gold Coast, Southport 4222 QLD, Australia

Email: joao.morimnascimento@griffithuni.edu.au 
This draft manuscript is distributed solely for purposes of scientific peer review. Its content is deliberative and predecisional, so it must not be disclosed or released by reviewers. Because the manuscript has not yet been approved for publication by the U.S. Geological Survey (USGS), it does not represent any official USGS finding or policy. 


\section{Introductory Paragraph}

Wind-waves are dominant contributors to coastal sea-levels ${ }^{1,2}$ and shoreline position ${ }^{3,4}$, and can be major disruptors of coastal population ${ }^{5,6}$, ecosystems ${ }^{7}$ and offshore/coastal structures. Understanding climate-driven impacts on the different variables of global wind-wave fields is critical to successful offshore/coastal climate adaptation planning ${ }^{4,8}$. Nonetheless, global wave-climate projections studies have strongly relied on single-method ensembles of limited size ${ }^{9}$, prohibiting a robust assessment of variations between wind-wave modelling approaches. Consequently, the uncertainty remains largely unquantified among existing projections of future wave climate ${ }^{9,10}$, which has subsequently hindered broad-scale assessments of future coastal risk and vulnerability ${ }^{11-14}$. Here, we present results from the first coherent, community-based multi-method ensemble of global wave-climate projections derived from ten independent state-of-the art studies, based on different dynamical and statistical approaches. Clustering of the ensemble members shows different spatial pattern characteristics of historical and projected future wave climate. Under a business-as-usual scenario, we find robust projected changes of $\sim 5-10 \%$ in annual mean significant wave height $\left(H_{s}\right)$, mean wave period $\left(T_{m}\right)$, and/or mean wave directions $\left(\theta_{m}\right)$ along $\sim 52 \%$ of the world's coastline (of which $\sim 22 \%$ exhibits robust change in two or more variables). Furthermore, we find that the variance in the existing community-ensemble is largely dominated by climate model-driven uncertainty, and that previous studies using single wind-wave modelling methods have been unable to resolve up to $~ 50 \%$ of the full variance associated with future wave-climate projections.

\section{Main body}

Anticipated changes to the wave climate will result from a combination of meteorologically-driven changes in near-surface ocean wind fields ${ }^{15}$ and morphologically-driven changes nearshore ${ }^{16}$, and can potentially exacerbate or exceed impacts of future projected sea-level rise $\mathrm{s}^{5,17,18}$. Establishing robust projections of global wave characteristics and quantifying the associated uncertainties introduced by the inherently complex modelling process is critical to avoid potentially costly maladaptation ${ }^{19}$. The wide range of wind-wave-modelling methods used to derive wave characteristics from surface wind fields expands the uncertainty space surrounding these projections, and remains poorly understood. The International Panel on Climate Change (IPCC) Fifth Assessment Report (AR5) ${ }^{20}$ assigned low confidence to wave projections (medium confidence for Southern Ocean $H_{S}$ increase) owing to the limited number of available model simulations and the uncertainty associated with Global Climate Model (GCM) downscaled surface wind fields. Since then, a new generation of global wave-climate projection studies have been completed by multiple international modelling groups ${ }^{21-30}$, using GCMforcing from the Fifth Coupled Model Intercomparison Project (CMIP5). While each of these studies have addressed aspects of uncertainty inherent to their own specific climate-modelling process, they treated the uncertainty space differently (emission scenarios and GCM-forcing) and none was able to quantify the uncertainty introduced by their own specific wind-wave modelling approaches (that is the different configurations of statistical/dynamical global wind-wave models) ${ }^{9}$. Consequently, there exist contrasting projected changes in wind-wave characteristics (magnitude and/or sign) across the world's oceans. These limitations have systematically hampered broad-scale assessments of future coastal risk 
and vulnerability which have either adopted future wave parameters obtained from a limited number of GCM-forced simulations surrounded by low confidence ${ }^{5}$; or have omitted any future changes ${ }^{13,14,31}$ on the basis of the unavailability of robust global data ${ }^{12}$ and the high uncertainty surrounding existing studies ${ }^{11}$.

Here, we perform a unique analysis of a coherent, multi-method ensemble of future global windwave climate scenarios derived from ten independent state-of-the-art studies ${ }^{21-30}$, which have been completed under a pre-established community-driven framework ${ }^{32,33}$. Together, such studies yield a grand ensemble of 148 members of global wave-climate projections, from which we identify robust projected meteorologically-driven changes in $H_{s}, T_{m}$ and $\theta_{m}$ at the global-scale. Further, we resolve the dominant sources of uncertainty (emission scenarios, model forcing and wind-wave modelling approaches) amongst these different projections of the world's future wave climate which has not been previously possible. Two ${ }^{29,30}$ of the ten contributing studies adopt statistical methods to derive wave projections exploiting a relationship between sea level pressure (SLP) fields and wind-wave parameters. The remaining contributions ${ }^{21-23,25-28,34}$ employ dynamical-based approaches in which GCM simulated high-temporal resolution surface winds are used to force a dynamical wave model. Details of each contribution and respective acronyms are provided in the Supplementary Information (Table S1).

Contributing studies have assessed the performance of each model to represent the historical wave climate on an individual basis. Here, we systematically evaluate the model skill of each contribution against the ERA-Interim ${ }^{35}$ (ERAI) global reanalysis wave data for the present time-slice (1979-2004) using spatial correlation (SC), normalized standard deviation (NSD) and centered-root-mean-square difference (CRMSD) (Supplementary Figs. S1-S3). Overall, both dynamical- and statistical-derived simulations exhibit good agreement relative to ERAI. CRMSD values in annual/seasonal $\bar{H}_{s}, \bar{T}_{m}$ and $\bar{\theta}_{m}$ are generally under $0.5 \mathrm{~m}, 0.5 \mathrm{~s}$ and $0.5^{\circ}$ respectively, and SC values are consistently above 0.9 . The statistically downscaled ECCC $(\mathrm{s})^{30}$ members and ERAI are not independent in the present timeslice, since these simulations have been obtained from a statistical-regression, calibrated using ERAIbased SLP and $H_{s}$ fields. Thus, both data sets are predisposed towards good agreement exhibiting SC and CRMSD values above 0.99 and under $0.20 \mathrm{~m}$, respectively, for all $H_{s}$ parameters. This is however not seen for the statistically downscaled IHC models which were calibrated using Global Ocean Wave (GOW2)-based SLP fields. Simulations from $\mathrm{IHC}^{29}$ and ECCC (s) ${ }^{30}$ share a common characteristic in which their members exhibit little variability relative to the dynamical projections, as a consequence of their statistical methods (Supplementary Fig. S1). Further discussion on model skill at seasonal and regional-scales is provided in Supplementary Information (Section 3.2; Fig. S2-S4).

Cluster analysis of $\bar{H}_{S}$ by model (Methods) over the representative present-day time-slice delineates groups of ensemble members that are strongly dependent on the wind-wave modelling approach used by each study to develop wave projections (Fig. 1). These results (supported by analysis of variations in wind-wave models and settings; Supplementary Information Section 3) show wave-modelling approach is the dominant source of variance within the ensemble of present-day wave simulations. There are two well-defined statistical-based clusters explained by differences in training data set and transfer functions, and three dynamical-based clusters arising from differences in wave modelling configurations, e.g. source-term physics. Statistical and dynamical-derived clusters exhibit differences 
in magnitude/spatial pattern (Fig. 1; Supplementary Fig. S5), emphasizing the dominant influence of wind-wave modelling approaches. A detailed discussion on the dissimilarities between simulations is provided in Supplementary Information, supported by global pairwise comparisons of mean and variability bias in a coherent subset with common forcing-method (Supplementary Table 3; Fig. S6S9).

Fig. 1 - Hierarchical clustering of annual mean significant wave height $\left(\bar{H}_{s}\right)$ for time-slice representing present-day climate (1979-2004). a, Cluster tree diagram (dendrogram) resulting from

Euclidean distance-based Ward's minimum variance clustering using global pairwise annual $\bar{H}_{s}$

(Methods). The vertical axis of the dendrogram represents the distance or dissimilarity between clusters (and cluster members) presented in log-scale for simplicity, higher lines are more dissimilar. In the horizontal axis of the dendrogram, the members are labelled by model forcing and study group (coloured accordingly). The multi-model ensemble mean from each group is also included with its respective colour. Multi-model ensemble averages (weighted multi-model mean by method, ENSEMBLE-WM, and uniformly weighted mean, ENSEMBLE) are coloured blue (Methods). The ERA-Interim is shown in green. Grey shading denotes five well-defined key clusters. b, Within each dashed line section, the cluster average of the relative difference to ERAI are shown for annual $\bar{H}_{S}$ (Methods). The numbers at the bottom left of each panel are the number of cluster members used to calculate the cluster average (ERAI was excluded).

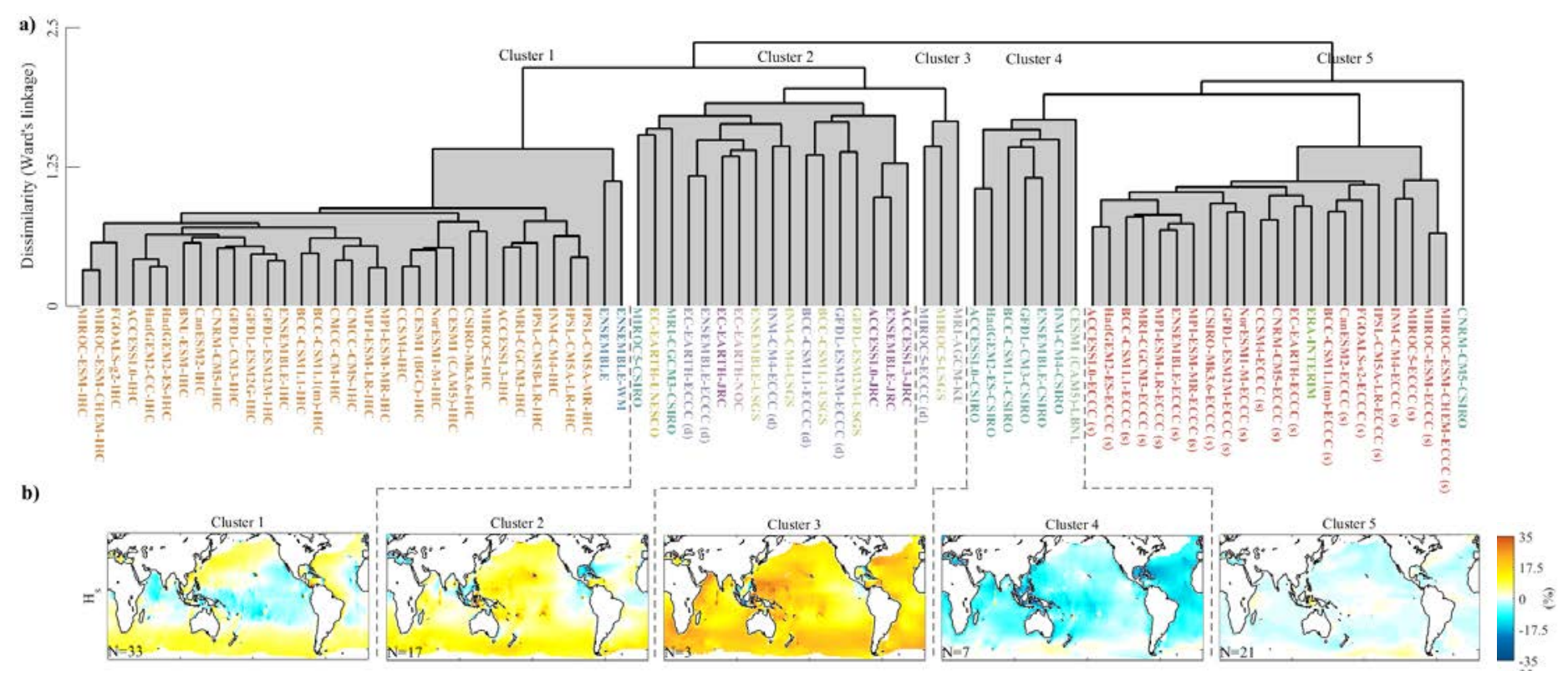


Future projected changes in global wave fields at the end of the $21^{\text {st }}$ century (2081-2100) are examined for two representative concentration pathways: an intermediate-emission (RCP4.5) and a high-emission scenario (RCP8.5). Changes in the weighted multi-model mean under RCP4.5 and RCP8.5 exhibit very similar spatial patterns for all wave parameters, but with relatively larger changes projected for RCP8.5. Signals of projected annual change in wave parameters $\left(\bar{H}_{s}, \bar{T}_{m}\right.$ and $\bar{\theta}_{m}$ ) exhibit agreement between models across $\sim 30 \%, 40 \%$ and $44 \%$ of global ocean under RCP8.5, respectively (Fig. 2; Supplementary Table S2).

A robust projected decrease in annual $\bar{H}_{S}$ is seen across the North Atlantic and portions of the northern Pacific Ocean of up to $10 \%$ under RCP8.5, expanding further across the eastern Indian and southern Atlantic Oceans in Austral summer. This is consistent with the relatively uniform decrease in projected surface wind speeds over the boreal extra-tropical storm belt ${ }^{36}$ partially driven by a strongly reduced meridional temperature gradient owing to polar amplification of climate change ${ }^{37}$. The regions of robust projected increase are limited to the Southern Ocean and the tropical eastern Pacific, in line with the intensification and poleward shift of the austral westerly storm belt ${ }^{38}$ and the increasing Southern Ocean swell propagation into the tropics ${ }^{10}$ respectively. In the Austral winter, regions of robust projected increase expand further across the tropics. These findings are (overall) qualitatively consistent with the Coordinated Ocean Wave Climate Project (COWCLIP) CMIP3based multi-model ensemble ${ }^{10}$ and other relevant literature ${ }^{9}$. Storm significant wave height $H_{s}^{99}$ exhibit similar annual and seasonal features of change as for $\bar{H}_{S}$, nevertheless the fraction of global ocean exhibiting robust changes is smaller (Fig. 2; Supplementary Table S2).

The extended influence of the increasing propagation of swells out of the Southern Ocean region into the tropics is shown by the robust projected increase in $\bar{T}_{m}$ (25\% of global ocean area) and the projected shift in $\bar{\theta}_{m}$ across $\sim 44 \%$ of the global ocean (clockwise in the tropical Pacific and tropical Atlantic and anticlockwise elsewhere). A further discussion on projected seasonal future changes and global pairwise comparison between the different contributions is provided in Supplementary Information (Section 4.1; Fig. S11-S14). The findings described above are mechanistically linked to well-documented large-scale atmospheric wind circulation changes ${ }^{36,37}$ and modes of natural climate variability ${ }^{10}$.

Beyond evaluating the robustness of projected changes in terms of inter-model agreement (Fig. 2), we assess the importance of the changes relative to the magnitude of the present-day inter-annual variability (Supplementary Fig. S10). For RCP4.5, and we speculate the same for lower pathways ${ }^{39}$, most robust projected changes in wind-wave characteristics fall within the range of present natural variability $(<100 \%)$. However, under the high-emission RCP8.5, nearly all robust changes exceed one standard deviation of the simulated inter-annual variability (some regions $>150 \%$ ). 
Fig. 2 - Projected future changes in weighted multi-model averaged wave-climate parameters under RCP4.5 and RCP8.5. a, Averaged weighted multi-model annual mean significant wave height $\bar{H}_{S}$, (December-February DJF and June-August JJA $\bar{H}_{S}$ within dashed box with same colorbar as for annual $\left.\bar{H}_{s}\right), 99^{\text {th }}$ percentile significant wave height, $H_{s}^{99}$, mean wave period, $\bar{T}_{m}$, and mean wave direction, $\bar{\theta}_{m}$, for the time-slice representing present climate (1979-2004). b-c, Averaged weighted multi-model changes for each respective wave parameter for the projected time-slice (2081-2100) relative to the present time-slice (\% change) under RCP4.5/RCP8.5, respectively. Changes in $\bar{\theta}_{m}$ are absolute changes with vector direction denoting $\bar{\theta}_{m}$ for the present-day time-slice. Hatching is applied to regions of robust change in which the magnitude of the climate signal is larger than the one standard deviation across the $n$-members of the weighted multi-model ensemble (methods). Seasonal changes for each wave parameter are provided in Supplementary Fig. S11-S12.

a) Historical (1979-2004)

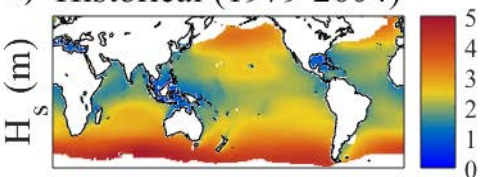

b) RCP4.5 (2081-2100)
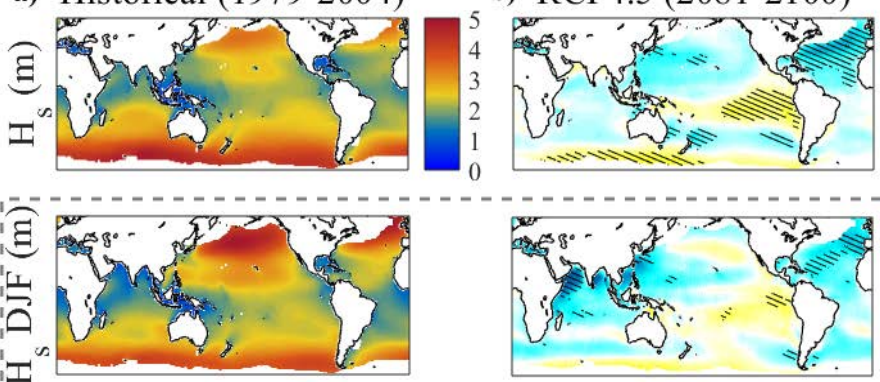

I
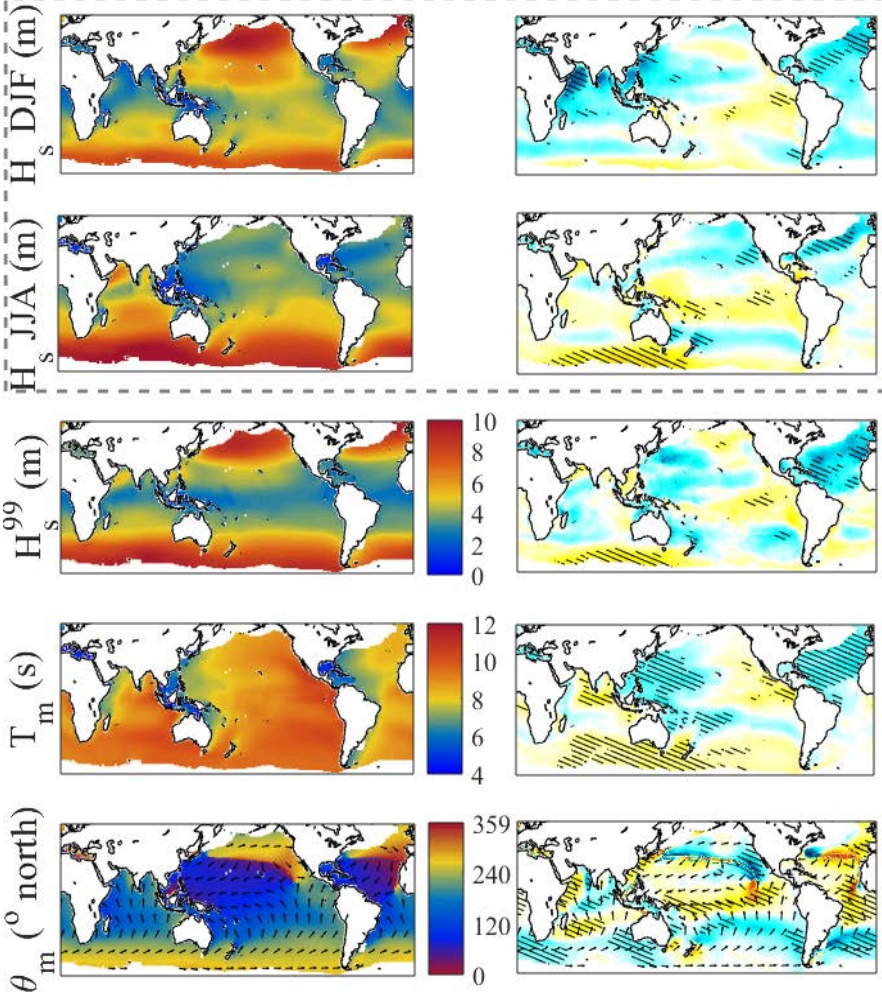
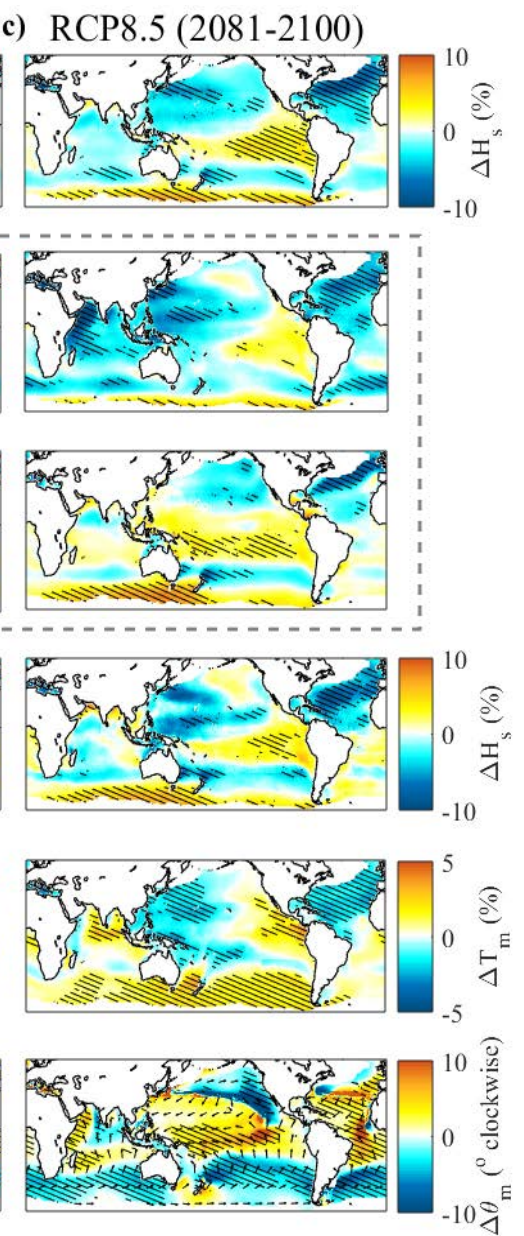
Fig. 3 presents the robust wave-climate projections (Fig. 2) in the context of the world's coasts, where impacts of any changes will be most acutely felt. Robust climate-driven changes in annual wave characteristics are projected across large sections of the world's coast with, $\sim 52 \%$ of coastline (excluding sea-ice areas and enclosed seas) exhibiting projected robust changes in at least one wave variable considered in this study (Fig. 3, Supplementary Table S2). Whilst there are areas where robust projections are limited to a single variable (e.g., $\bar{\theta}_{m}$ changes on the southern and eastern coasts of Africa), there are several coastal sections ( $22 \%$ of the world's coast) where robust projections in $\bar{H}_{s}, \bar{T}_{m}$ and/or $\bar{\theta}_{m}$ coincide (e.g., New Zealand, Southern Australia or Chile). This is also the case for the highly-vulnerable Pacific/Indian Ocean low-lying countries ${ }^{6}$ and the highly populated North American Atlantic coast which is a well-documented 'hotspot' of accelerated sea-level rise ${ }^{40}$. Future projected changes in $\bar{\theta}_{m}$ (a key driver of erosion patterns ${ }^{8,41}$ and island morphodynamic changes ${ }^{42}$ ) are robust along many coastlines with magnitudes of change ranging between $\sim \pm 17^{\circ}$. 
Fig. 3 - World's coastlines exhibiting projected robust changes in significant wave height $\left(\overline{\boldsymbol{H}}_{\mathrm{s}}\right)$, period $\left(\bar{T}_{m}\right)$ and direction $\left(\overline{\boldsymbol{\theta}}_{m}\right)$ by 2080-2100 under RCP8.5. Sections of coast exhibiting robust weighted multi-model mean changes under RCP8.5 are coloured according to the qualitative colourbar (bottom), which also shows the respective percentage of coast where changes are robust (Methods for definition) for each wave characteristic(s) under RCP8.5 (top line) and RCP4.5 (bottom line). Sections of coast exhibiting a simultaneous robust increase in $\bar{H}_{\mathrm{s}}$ and robust decrease in $\bar{T}_{\mathrm{m}}$ (or vice versa) are extremely limited. Vectors represent sections of coast with projected robust $\bar{\theta}_{\mathrm{m}}$ changes with their angle $\left({ }^{\circ}\right.$ north) representing wave direction over the representative time-slice (1979-2004) and their color representing the magnitude of the future changes (according to the quantitative colourbar, right side). The percentage of the world's coast with robust changes in $\bar{\theta}_{m}$ is estimated at 29\% (Supplementary Table S2). Coasts without black outline represent sea-ice areas and enclosed seas excluded from analysis (Methods).

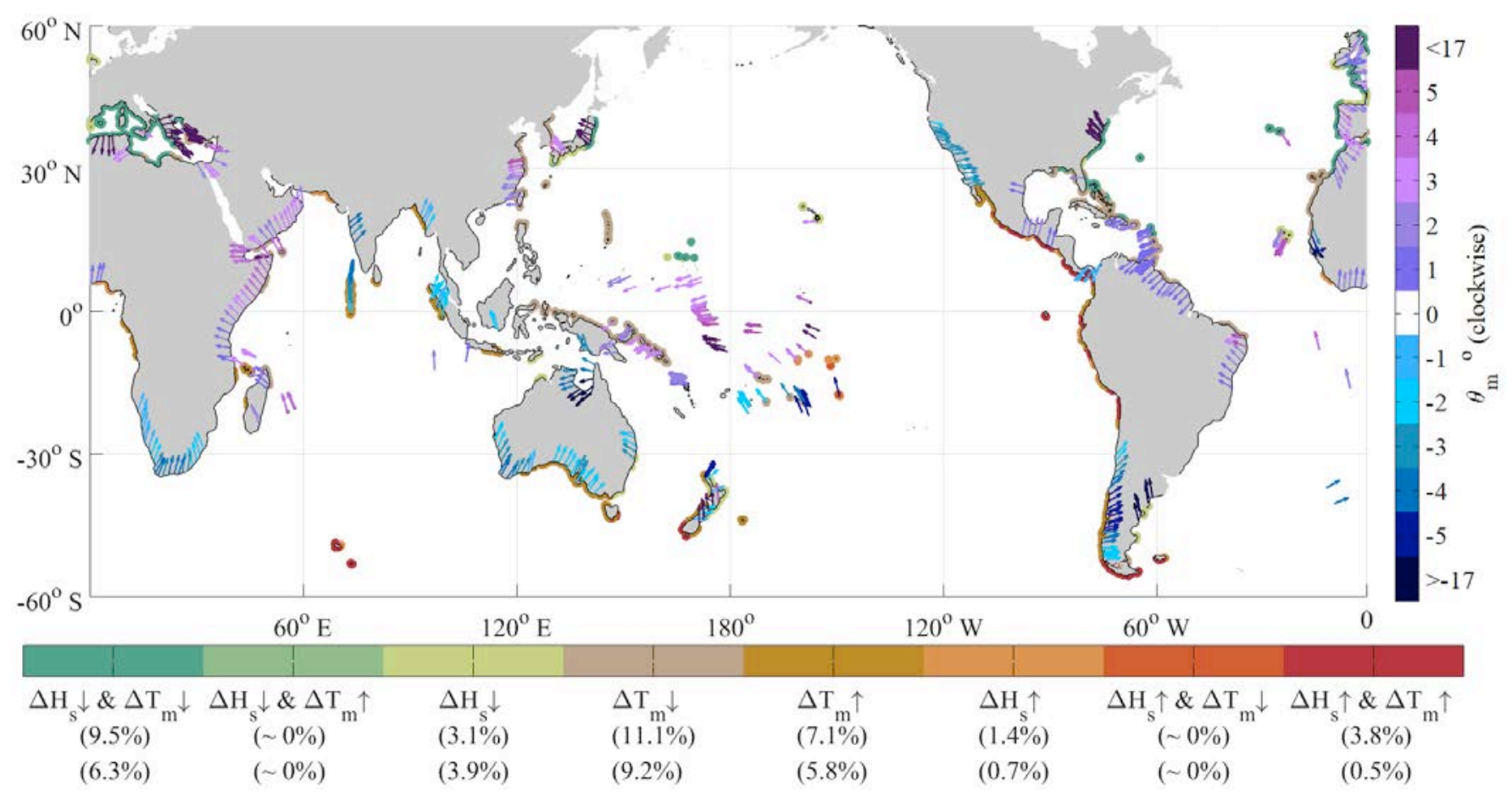


The community ensemble of wave climate projections exhibits a range of uncertainty stemming from several different sources (emission scenarios, RCPs; model forcing, GCMs; and wind-wave modelling methods, WMs) which have been largely unquantified in previous studies. We applied Ward's ANOVA-based clustering (Methods) to a designed subset of projection scenarios (consult Supplementary Table S3) spanning 2 RCP scenarios, 10 forcing GCMs and $10 \mathrm{WMs}$, providing an overall analysis of the dissimilarity amongst the projected signals (Fig. 4; Supplementary Fig. S16). We find that climate change signals largely cluster by GCM-forcing (wind forcing from which the wave field originates). There are some cases where RCP- and/or WM-related uncertainty dominate dissimilarity among the simulations (e.g., MIROC5, BCC-CSM1.1 and MRI-CGCM3). Analysis of the full ensemble of projections shows consistent results but less simply interpreted (Supplementary Fig. S16).

To further elucidate the dominant drivers of variance amongst these wave projections (and their relative contribution), we applied a three-factor ANOVA (Methods) to a set of coherent ensembles available as subsets of the full ensemble (Supplementary Table S4). The results show that no source of variance is negligible, and that projection uncertainty is not solely attributable to the different sources of uncertainty but also depend upon their nonlinear interactions. For each of the subsets analyzed (Fig. 5; Supplementary Fig. S17-S19), we find a dominating influence of GCM uncertainty across most of the global ocean accounting for $\sim 30 \%$ to over $50 \%$ of the total variance associated with the projected change signal of $\bar{H}_{S}$ (consistent with our cluster analysis; Fig. 4). Scenario uncertainty dominates across the North Atlantic, western North Pacific Ocean and Southern Ocean ( 40 to more than $50 \%$ of the total variance), but is exceeded by other uncertainty contributors elsewhere. These results are consistent, to a certain extent, with previous research using statistical ensembles ${ }^{29,43}$ which found GCM-driven uncertainty to be larger than scenario uncertainty. However, these studies report that scenario uncertainty only accounts for less than $5 \%$ of the total variance anywhere, whereas our results demonstrate that its contribution is much larger across the afore-mentioned regions. Windwave modelling method is a considerable contributor to the total uncertainty particularly across the tropics and subtropics ( $25-50 \%$ of the total variance), whilst nonlinear interactions contribute $\sim 20$ $30 \%$ of the total uncertainty over most of the global ocean (dominated by interactions between GCMWM) (Fig. 5). These results show that all three sources of variance have to be systematically sampled to capture the total uncertainty in the projected change signal. It also indicates previous studies using a single WM have not captured up to $40-50 \%$ of the total variance (sum of the fractions attributable to WMs; Fig. 5). 
Fig. 4 - Hierarchical clustering of projected future changes in annual mean significant wave height $\left(\overline{\boldsymbol{H}}_{s}\right)$ (2081-2100 relative to 1979-2004). a, Cluster dendrogram resulting from Euclidean distance-based Ward's minimum variance clustering using global pairwise projected change annual $\bar{H}_{s}$ (Methods). The vertical axis of the dendrogram represents the distance or dissimilarity between clusters (and cluster members) presented in log-scale for simplicity. In the horizontal axis of the dendrogram, the members are labelled by model forcing, study group and RCP scenario (RCP4.5 simulations are italicized) respectively, and coloured by GCM, accordingly. The multi-model ensemble mean from each study group is also included. Multi-model ensemble averages (weighted multi-model mean by method, ENSEMBLE-WM, uniformly weighted mean, ENSEMBLE, and weighted multi-model mean by forcing, ENSEMBLE-WF) are coloured blue (methods). Grey shading denotes seven well-defined key clusters. $\mathbf{b}$, Within each dashed line section, cluster-averaged projected changes in annual $\bar{H}_{S}(\mathrm{~m})$ between are shown (Methods). The numbers at the bottom left of each panel are the number of cluster members used to calculate the cluster average.

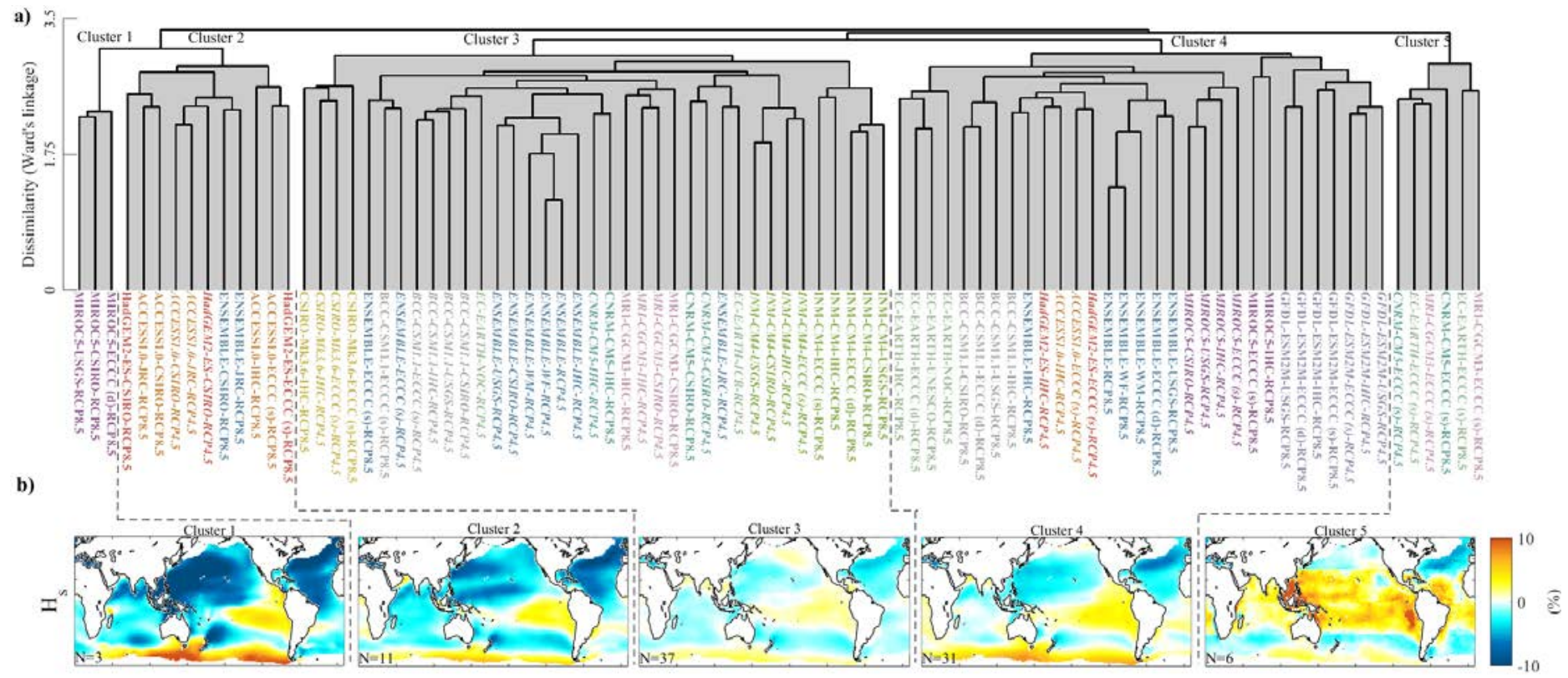


Our study does not address variance in projections of wave climate fields introduced with atmospheric downscaling techniques. Although the regional downscaling step has been widely used in wave climate projection studies and is a topic of intensive research ${ }^{44}$, the different downscaling techniques introduce an additional source of variance which (at present) is not possible to sample at the global-ocean scale. We also note that the ensemble subsets suitable for ANOVA are limited relative to the full community ensemble. Nonetheless, the GCM-forcing within each subset represents a broad cross-section of the full CMIP5 ensemble with available high-temporal resolution surface wind forcing ${ }^{21,45}$, model components, spatial resolution and skill ${ }^{45}$. 
Fig. 5 - Relative contribution of different sources of uncertainty to projected future changes in mean significant wave height $\left(\overline{\boldsymbol{H}}_{\boldsymbol{s}}\right)$. a-d, Fraction of the total variance in annual $\bar{H}_{s}$ changes (20812100 relative to 1979-2004) attributable to a) global climate models (GCMs), b) wind-wave modelling methods (WMs), c) greenhouse-gas emission pathways (RCPs) and $\mathbf{d}$ ) sum of pairwise nonlinear interactions. e) Spatially-averaged contribution of each uncertainty source and their

pairwise nonlinear interactions to the total ensemble uncertainty. Note the relatively small contribution of model residuals. Results are derived from ensemble subset 2 which comprises 6 GCMs, 2 RCPs and 3 WMs for a total of $N=36$ simulations (Supplementary Table S4). Similar results for subset 1 and 3 are presented in Supplementary Fig. S16-S17. Variance partitioning based on a three-factor ANOVA complemented with a subsampling scheme (Methods). Note that plotting artifacts such as horizontal lines reflect the effects of the spatial-domain partition applied in statistical methodologies.
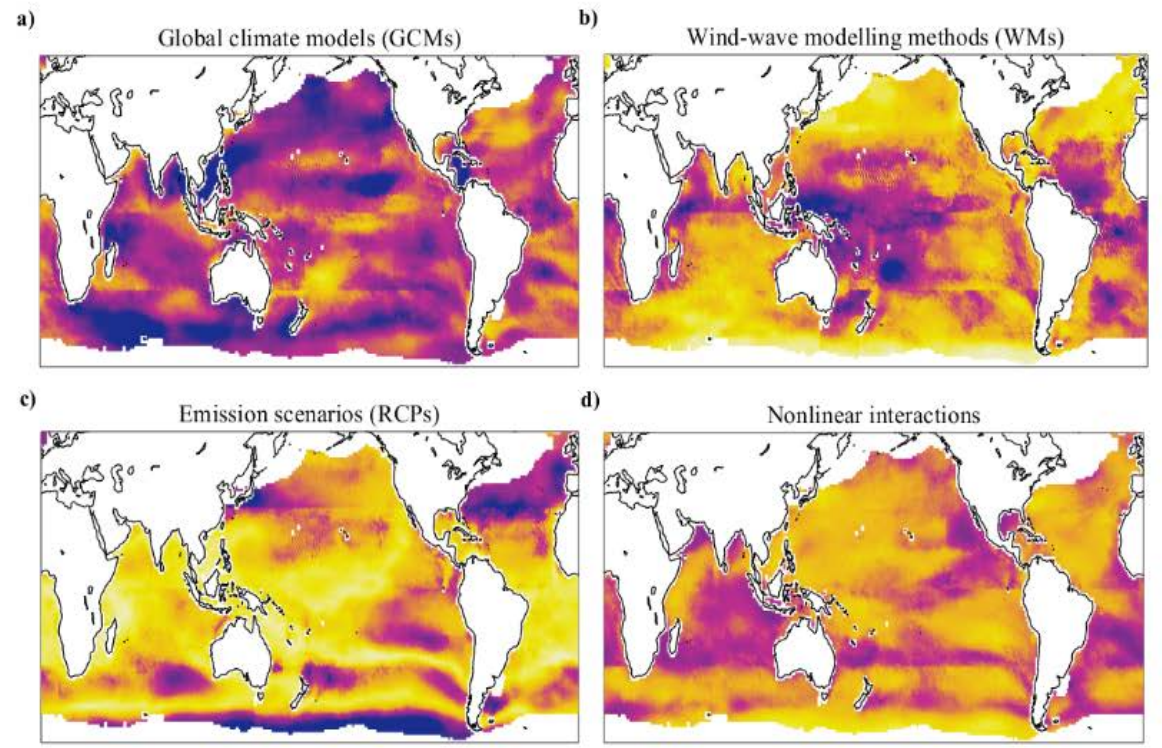

Fraction of total variance

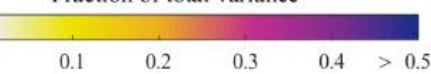

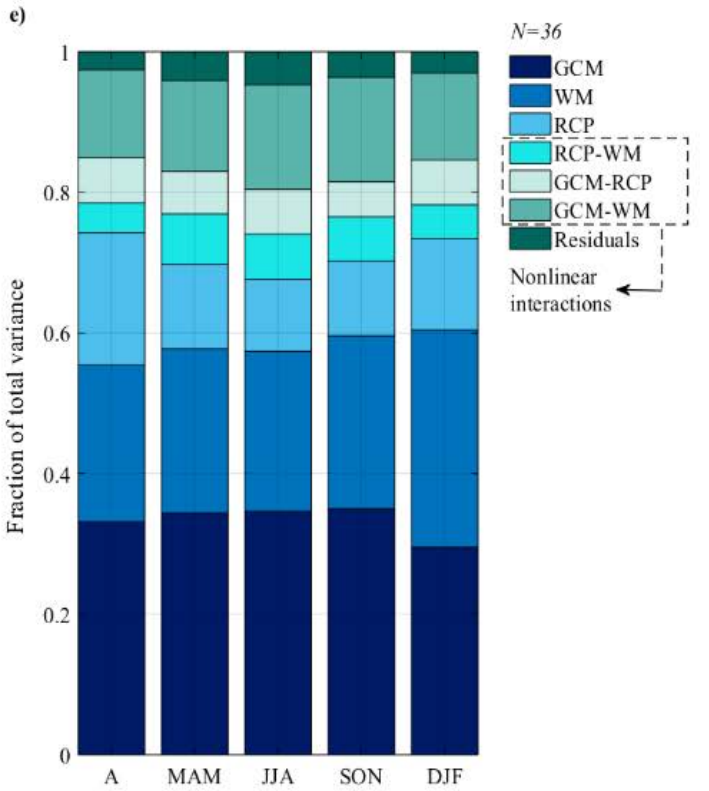


Our CMIP5-derived coherent ensemble of wave-climate projections samples across RCPs, GCMs and WMs, allowing a much improved systematic sampling of the uncertainty space compared to the COWCLIP CMIP3-based ensemble of opportunity ${ }^{10}$ and/or any previous study to date. In addition to resolving the largely unquantified contribution of the dominant sources of uncertainty, it attests to the importance of using conceptually different WMs (similar to water-scarcity projections ${ }^{46}$ ). Some of the existing variability among dynamical WMs caused by $H_{s}$ biases may be potentially reduced by further model calibration ${ }^{47}$. While at present it impossible to separate this component, we advocate that future dynamical wind-wave simulations attempt to reduce the overall $H_{s}$ bias. These findings also highlight the need to understand how different available global wind-wave reanalysis/hindcasts (used to derive historical trends of wave climate change ${ }^{1}$ ) differ. We need to understand whether the same level of variance lies between these products. Our findings provide a new perspective on the robustness of global multivariate wave projections which builds beyond the restricted range of future wave-climate scenarios published to date. These coordinated ensemble projections show signals of wave climate change will not exceed the magnitude of the internal climate variability if the goal of the Paris Agreement $2^{\circ} \mathrm{C}$ degree target is kept. Under a business-as-usual scenario, $\sim 52 \%$ of the world's coastline will endure robust annual changes in $H_{s}, T_{m}$ and/or $\theta_{m}$ (with $\sim 22 \%$ exhibiting robust changes in at least two of these variables). The magnitude of the projected future changes in any of these wave variables ( 5-10\%) are capable of inducing notable changes in coastal wave-driven processes and associated hazards ${ }^{41}$. While our results have far-reaching implications from many perspectives, they only address meteorologically-driven changes in wind-wave characteristics, which have been the predominant focus of wind-wave climate projection studies to date. Some localised studies suggest the morphologically-driven component of wave climate change (induced by rising sea-level, reef stability and other beach morphology changes) might lead to greater change in the coastal zone than these meteorologically-driven changes ${ }^{16}$. Concentrated community effort is now required to quantify morphologically-driven wave climate change as a contributor to global coastal sea-level change, as we look towards improved coastal vulnerability assessments from the climate community ${ }^{48}$. 


\section{Methods.}

\section{Data contribution}

We use a community-derived ensemble compiled from ten CMIP5-derived global wind-wave climate projection studies ${ }^{21-30}$ completed under a pre-established designed framework ${ }^{32,33}$. Annual and seasonal means of significant wave height $\left(H_{s}\right)$, mean wave period $\left(T_{m}\right)$ and mean wave direction $\left(\theta_{m}\right)$ as well as $10^{\text {th }}$ and $99^{\text {th }}$ percentiles of annual/seasonal $H_{s}$ are obtained from the ten contributions (Supplementary Information for detailed description of data sets and framework). Our analysis assesses projected $21^{\text {st }}$ century changes between the present-day (1979-2004) and future (2081-2100) time-slices. These align with CMIP5 archives of high-temporal resolution wind data required for wave-model forcing, and correspond to the common period across nine of the ten contributing data sets. Contributed wave data has been considered under two greenhouse-gas concentration representative pathways: RCP4.5 and RCP8.5 characterizing medium-stabilizing and high-radiative forcing (reaching $+4.5 \mathrm{~W} / \mathrm{m}^{2}$ and $8.5 \mathrm{~W} / \mathrm{m}^{2}$ relative to pre-industrial 1850 -conditions), respectively. Sea-ice ocean areas were excluded from this analysis to support intercomparison between the different contributions.

\section{Model-skill analysis}

Model skill for the present-day wave climate (1979-2004) was assessed by comparison with the wave parameters obtained from the ERA-Interim (ERAI) global wave reanalysis (Supplementary Information). To allow for intercomparison, the wave parameters derived from each of the global wave data sets were bilinearly interpolated onto the ERAI grid at $1^{\circ}$ spatial resolution. Taylor diagrams were used to compare model performance for each wave parameter at global- and regionalscales. The global model-skill analysis and regional evaluation performed over 10 sub-domains of the global ocean is provided in Supplementary Information.

\section{Cluster methodology}

We applied an agglomerative-hierarchical clustering analysis, with the similarity criterion defined by Ward's ANOVA-based minimum variance algorithm ${ }^{49}$. Initial cluster distances were determined using a multi-dimensional approach, where the pair-wise Euclidean distance $(D)$ amongst ensemble members are calculated at every grid node (rather than spatially-averaged), thereby clustering members with high similarity in terms of spatial pattern and magnitude:

$$
D_{i, j, k}=\sqrt{\sum_{k=1}^{w}\left(x_{i, k}-x_{j, k}\right)^{2}}
$$

where $x_{i, k}$ and $x_{j, k}$ are the magnitudes of the relative projected change in the annual mean significant wave height from models $i$ and $j$ respectively, at grid point $k$, with $w$ equal to the number of ocean grid points. Note that for the clustering of present-day wave simulations, we used absolute values rather than relative changes. The usage of the annual mean significant wave height $\left(\bar{H}_{S}\right)$ as our clustering variable is based on the fact that $\bar{H}_{S}$ is the only parameter available from all contributions, 
and our main goal is to analyse the whole community ensemble of wave simulations. Note that statistical-method-derived members ${ }^{30,50}$ do not provide wave period and/or directions due to methodological constraints (Supplementary Table S1). To further support our results, we performed a multivariate clustering based on annual $\bar{H}_{s}, \bar{T}_{m}$ and $\bar{\theta}_{m}$ using our dynamical subset exhibiting qualitatively similar results in the present-day simulations and future relative changes. Further description of the cluster application to the present-day climate and the future projected changes is provided below.

\subsection{Application to present-day simulations}

Annual $\bar{H}_{S}$ over the present time slice (1979-2004) was used in the clustering procedure. We included all ensemble members available as well as the ensemble average of each contributing study, a uniformly weighted multi-model mean (attributing equal weight to each individual member) and a weighted multi-model mean by method (or approach). The latter consisted of reducing the full ensemble to $n$-members, with each single member representing the mean from a given study (when available). For example, the 30-member IHC ensemble was reduced to one member representing its multi-model mean. The relative differences $(\%)$ between the average of all members within each main cluster (when available) and the ERAI data was calculated for each parameter analysed over the global ocean (Fig. 1, and Supplementary Fig. S5). We extended our clustering analysis to annual and seasonal $\bar{H}_{S}$ values combined and the results were consistent with those obtained using annual mean values. We also applied the clustering approach to the other wave parameters individually obtaining consistent results. In both cases, present simulations are strongly dependent on the method taken by each study group to develop future wave fields as shown in Fig. 1.

\subsection{Application to future projected changes}

To identify and resolve similarities in the projected future change signal, the clustering procedure was applied to the projected changes in annual $\bar{H}_{S}$ between present-day (1979-2004) and future (20812100) time-slices. To resolve the relative importance of the different sources of variance (RCP scenario, GCM forcing and wave-modelling methods), we selected a subset from the full ensemble where each member shares common GCM forcing with at least two other members (obtained from different wave-modelling approach) (Supplementary Table S2). In the clustering procedure, we included the ensemble average of each contribution, a uniformly weighted multi-model mean (section 3.1), a weighted multi-model mean by climate-model forcing (section 5) and a weighted multi-model mean by method, for each RCP. Five main clusters were identified based on the clustering results as indicative of members with considerable dissimilarity in the projected change signal. The average of all members within each main cluster (when available) was calculated for each wave parameter (Fig. 1; Supplementary Fig. S5), providing a robust indication of spatial and magnitude dissimilarities across the global ocean.

We further applied the ANOVA-based clustering algorithm to the full community ensemble of wave projections, presenting consistent dissimilarities and respective associations between all available wave simulations, albeit less clear owing to the large size of the ensemble (Supplementary Fig. S15). 


\section{ANOVA method}

\subsection{Approach and selection of subsets}

Variance across projected future wave climate changes (2081-2100 relative to 1979-2004) within our community-based ensemble arises from three different sources: choice of emission scenarios (RCPs), global climate models (GCMs), and wind-wave modelling methods (WMs). The latter refers to different configurations of the statistical/dynamical wind-wave models used to derive future global wave fields. In contrast with other climatic variables (e.g., temperature or precipitation), dynamicallyderived multi-model ensembles of wave projections are usually only available for twenty-year timeslices over which high-temporal resolution GCM-derived near-surface wind fields are available ${ }^{9,33}$ (Supplementary Table S2), prohibiting any partitioning of projection uncertainty in time. Therefore, we decompose the total ensemble uncertainty into contributions from the different sources of uncertainty (RCPs, GCMs and WMs) and the nonlinear interactions between them, where the total ensemble uncertainty is the variance of the climate change signal in projected average annual/seasonal $\bar{H}_{S}$. The fraction of variance attributable to each source at each grid point was determined based on a three-factor ANOVA ${ }^{51}$ (section 4.3). The ANOVA was applied to three subsets out of the full ensemble individually, each with a distribution of simulations sharing our three sources of uncertainty (Supplementary Table S3).

\subsection{Subsampling scheme}

Traditional applications of ANOVA using different sample sizes of variance sources result in biased variance estimators $^{52}$ (cf. Fig. 4 and Supplementary Fig. S15-16 with Supplementary Fig. S17). In order to reduce the effect of the biased variance estimator on the quantification of the uncertainty contribution we complemented the ANOVA with a subsampling scheme previously proposed ${ }^{52}$. In each subsampling iteration $i$, we select two of $n$-climate models and two of $m$-wave models, representing a total of $C_{2}^{n} C_{2}^{m}$ subsamples with $n$ and $m$ denoting the number of GCMs and WMs within each subset respectively. For each subsample $i$, we ended up with two global climate models, two emission scenarios and two wavemodelling approaches which we used for variance decomposition as described below.

\subsection{Three-factor ANOVA model}

Letting $Y_{j k l}^{i}$ be our response variable representing the projected change signal in $\bar{H}_{s}$ from the $j^{\text {th }}$ GCM, $k^{\text {th }} \mathrm{RCP}$ and $l^{\text {th }} \mathrm{WM}$, we define our three-factor ANOVA model without replication following $^{52,53}$ :

$$
Y_{j k l}^{i}=\mu^{i}+\alpha_{j}^{i}+\beta_{k}^{i}+\gamma_{l}^{i}+(\alpha \beta)_{j k}^{i}+(\alpha \gamma)_{j l}^{i}+(\beta \gamma)_{k l}^{i}+\delta_{j k l}^{i}
$$

where $\mu^{i}$ is the grand-mean projected change of the subsample $i$. The terms $\alpha_{j}^{i}, \beta_{k}^{i}$ and $\gamma_{l}^{i}$ represent the variance attributable to the different factors (GCM, RCP and WMs respectively) with $j, k$ and $l$ denoting samples of the different factors. The terms $\alpha \beta_{j k}^{i}, \alpha \gamma_{j l}^{i}$ and $\beta \gamma_{k l}^{i}$ represent nonlinear interactions between the different pairs of factors, respectively. The term $\delta_{j k l}^{i}$ represents the variance attributable to residuals of the model (which are assumed to be normally distributed having zero- 
average and variance $\sigma_{\text {res }}^{2}$ ) and internal variability. The results derived from each subsample $i$ for each subset were used to estimate the unbiased fraction of the total uncertainty attributable to each source ${ }^{52,53}$. The nature of our ensemble allows us to quantify the contribution of each source of uncertainty, and their first-order interactions, to the total variance, but does not allow us to test whether these stand out of the natural internal variability of the climate system or resolve GCM generated internal variability. An analysis of the climate change signal relative to the amplitude of the internal climate variability is provided in Supplementary Fig. S10 based on one realisation for each member.

\section{Analysis of projected change}

Changes in all wave variables (except $\bar{\theta}_{m}$ ) between the present and future time-slices were derived as percentage changes for each member (from each contribution) directly forced by GCM-derived surface wind fields. Both $\mathrm{LBNL}^{27}$ and $\mathrm{KU}^{28}$ data sets were derived using downscaled forcing via high resolution atmospheric models driven by specific SST conditions (Supplementary Information) and therefore were not included in this analysis. Projected changes in $\bar{\theta}_{m}$ were calculated as absolute values and shown as clockwise, or anticlockwise, rotation in degrees relative to the present-day climate mean. Changes were calculated under RCP4.5 and RCP8.5. A multi-model weighted mean projected change was calculated. Fifty statistical projected scenarios are available from IHC and ECCC (s) combined for both scenarios, whereas dynamical projections consist of 23 (RCP4.5) and 25 (RCP8.5) projected wave-climate change scenarios (Supplementary Table S1). Projected change signals strongly depend on GCM forcing (the surface wind fields from which the wave field originates from) (Fig. 3 and 4), hence a weighted multi-model ensemble average was calculated by applying a weighting factor to each member:

$$
\bar{x}_{k}=\frac{\sum_{i=1}^{n}\left(\Delta_{i, k} \times W_{i, k}\right)}{\sum_{i=1}^{n}\left(W_{i, k}\right)}
$$

where $\Delta_{i, k}$ is the projected change for a given wave parameter $k$ by the ensemble member $i$ and $W_{i}$ is the weighting factor for the ensemble member $i$ for that same parameter (calculated as the number of ensemble members with that same forcing GCM amongst all members $n$ ). For all wave parameters, the map of mean projected change was derived as the $n$-member multi-model weighted mean difference between projected and present wave-climate fields from Equation 3. Stippling is shown where the multi-model weighted mean response exceeds the model spread (i.e. a robust change), measured as the weighted standard deviation across the $n$-members:

$$
S_{k}=\sqrt{\frac{\sum_{i=1}^{n} W_{i, k}\left(\Delta_{i, k}-\bar{x}_{k}\right)}{\sum_{i=1}^{n}\left(W_{i, k}\right)-1}}
$$

\section{Percentage of coastline with robust changes}

For annual and seasonal means of each wave parameter, the percentage of global coastline length that exhibits a robust, by the above definitions (section 5), projected increase/decrease was calculated (Table S1). To this end, we used the nearest model deep-water grid-point to the coastline data set from the Global Self-consistent Hierarchical High-resolution Geography (GSHHG) archive ${ }^{54}$. Coasts 
without available wave model outputs were not considered, which included sea-ice regions and enclosed seas. These results are considered representative of wind-wave conditions before interaction with the seafloor and depth-induced wave breaking in the nearshore occurs ${ }^{55}$. 


\section{Acknowledgements.}

This study represents Task 3 of the second phase of the Coordinated Ocean Wave Climate Project (COWCLIP) (https://cowclip.org/), an international collaborative working group endorsed by the Joint Technical Commission for Oceanography and Marine Meteorology (JCOMM) - a partnership between the World Meteorological Organization (WMO) and the Intergovernmental Oceanographic Commission of UNESCO (IOC-UNESCO). We acknowledge the different climate modelling groups, the Program for Climate Model Diagnosis and Intercomparison (PCMDI) and the World Climate Research Program's (WCRP) Working Group on Coupled Modelling (WGCM). J.M acknowledges the support of Griffith University School of Engineering and Built Environment and the Australian National Environmental Science Program (NESP). M.H. and C.T. acknowledge the support of NESP Earth Systems and Climate Change Hub. B.T and M.W acknowledge the support of the Regional and Global Climate Modeling Program of the U.S. Department of Energy, Office of Science, Office of Biological and Environmental Research (contract number DE-AC0205CH11231) and the National Energy Research Supercomputing Center (NERSC) of the Lawrence Berkeley National Laboratory. L.E. acknowledges the support of the US Geological Survey Coastal and Marine Hazards/Resources Program. We thank all contributors to the COWCLIP project including Alberto Meucci (University of Melbourne, Australia), Christian Appendini (National Autonomous University of Mexico, Mexico), Fabrice Ardhuin (Ifremer, France), Nikolaus Groll (Helmholtz-Zentrum Geesthacht Zentrum, Germany), Sarah Gallagher (Met Éireann, Ireland), Sergey Gulev (Moscow State University, Russia) and Will Perrie (Bedford Institute of Oceanography, Canada). 


\section{References.}

1 Melet, A., Meyssignac, B., Almar, R. \& Le Cozannet, G. Under-estimated wave contribution to coastal sea-level rise. Nature Climate Change 8, 234-239, doi:10.1038/s41558-018-0088-y (2018).

2 Katherine, S., Peter, R. \& Hilary, S. The relative contribution of waves, tides, and nontidal residuals to extreme total water levels on U.S. West Coast sandy beaches. Geophysical Research Letters 44, 1839-1847, doi:doi:10.1002/2016GL071020 (2017).

3 Harley, M. D. et al. Extreme coastal erosion enhanced by anomalous extratropical storm wave direction. Scientific Reports 7, 6033, doi:10.1038/s41598-017-05792-1 (2017).

$4 \quad$ Barnard, P. L. et al. Extreme oceanographic forcing and coastal response due to the 20152016 El Niño. Nature Communications 8, 14365, doi:10.1038/ncomms 14365 https://www.nature.com/articles/ncomms14365\#supplementary-information (2017). Storlazzi, C. D. et al. Most atolls will be uninhabitable by the mid-21st century because of sea-level rise exacerbating wave-driven flooding. Science Advances $\mathbf{4}$, doi:10.1126/sciadv.aap9741 (2018).

6 Hoeke, R. K. et al. Widespread inundation of Pacific islands triggered by distant-source wind-waves. Global and Planetary Change 108, 128-138, doi:https://doi.org/10.1016/j.gloplacha.2013.06.006 (2013).

7 Feagin, R. A. et al. Does vegetation prevent wave erosion of salt marsh edges? Proceedings of the National Academy of Sciences 106, 10109-10113, doi:10.1073/pnas.0901297106 (2009).

8 Barnard, P. L. et al. Coastal vulnerability across the Pacific dominated by El Niño/Southern Oscillation. Nature Geoscience 8, 801, doi:10.1038/ngeo2539 https://www.nature.com/articles/ngeo2539\#supplementary-information (2015).

9 Morim, J., Hemer, M., Cartwright, N., Strauss, D. \& Andutta, F. On the concordance of 21st century wind-wave climate projections. Global and Planetary Change 167, 160-171, doi:https://doi.org/10.1016/j.gloplacha.2018.05.005 (2018).

10 Hemer, M. A., Fan, Y., Mori, N., Semedo, A. \& Wang, X. L. Projected changes in wave climate from a multi-model ensemble. Nature Climate Change $\mathbf{3}, 471$, doi:10.1038/nclimate1791 https://www.nature.com/articles/nclimate1791\#supplementary-information (2013).

11 Arkema, K. K. et al. Coastal habitats shield people and property from sea-level rise and storms. Nature Climate Change 3, 913, doi:10.1038/nclimate1944 https://www.nature.com/articles/nclimate1944\#supplementary-information (2013).

12 Wahl, T. et al. Understanding extreme sea levels for broad-scale coastal impact and adaptation analysis. Nature Communications 8, 16075, doi:10.1038/ncomms16075 https://www.nature.com/articles/ncomms16075\#supplementary-information (2017).

13 Hinkel, J. et al. Coastal flood damage and adaptation costs under 21st century sea-level rise. Proceedings of the National Academy of Sciences 111, 3292-3297, doi:10.1073/pnas.1222469111 (2014).

14 Vitousek, S. et al. Doubling of coastal flooding frequency within decades due to sea-level rise. Scientific Reports 7, 1399, doi:10.1038/s41598-017-01362-7 (2017).

15 Hemer, M. A., Katzfey, J. \& Trenham, C. E. Global dynamical projections of surface ocean wave climate for a future high greenhouse gas emission scenario. Ocean Modelling 70, 221245, doi:https://doi.org/10.1016/j.ocemod.2012.09.008 (2013).

16 Oliveira, F. S. B. F. A Case Study of Wave Climate Changes due to Nearshore Morphological Evolution. Journal of Coastal Research 24, 21-32 (2008).

17 Coelho, C., Silva, R., Veloso-Gomes, F. \& Taveira-Pinto, F. Potential effects of climate change on northwest Portuguese coastal zones. ICES Journal of Marine Science 66, 14971507, doi:10.1093/icesjms/fsp132 (2009). 

Based Design of Caisson Breakwaters. Journal of Waterway, Port, Coastal, and Ocean Engineering 138, 215-225, doi:10.1061/(ASCE)WW.1943-5460.0000126 (2012).

19 Kunreuther H., S. G., V. Bosetti, R. Cooke, V. Dutt, M. Ha-Duong, H. Held, J. LlanesRegueiro, A. Patt, E. Shittu, and E. \& Weber. Integrated Risk and Uncertainty Assessment of Climate Change Response Policies. In: Climate Change 2014:

Mitigation of Climate Change. Contribution of Working Group III to the Fifth Assessment Report of the Intergovernmental Panel on Climate Change [Edenhofer, O., R. Pichs-Madruga, Y. Sokona, E. Farahani, S. Kadner, K. Seyboth, A. Adler, I. Baum, S. Brunner, P.

Eickemeier, B. Kriemann, J. Savolainen, S. Schlömer, C. von Stechow, T. Zwickel and J.C. Minx (eds.)]. (Cambridge, United Kingdom and New York, NY, USA, 2014).

20 Church, J. A., P.U. Clark, A. Cazenave, J.M. Gregory, S. Jevrejeva, A. Levermann, M.A. Merrifield, G.A. Milne, R.S. \& Nerem, P. D. N., A.J. Payne, W.T. Pfeffer, D. Stammer and A.S. Unnikrishnan. Sea Level Change. In: Climate Change 2013: The Physical Science Basis. Contribution of Working Group I to the Fifth Assessment Report of the Intergovernmental Panel on Climate Change [Stocker, T.F., D. Qin, G.-K. Plattner, M. Tignor, S.K. Allen, J. Boschung, A. Nauels, Y. Xia, V. Bex and P.M. Midgley (eds.)]. (Cambridge, United Kingdom and New York, NY, USA., 2013).

21 Hemer, M. A. \& Trenham, C. E. Evaluation of a CMIP5 derived dynamical global wind wave climate model ensemble. Ocean Modelling 103, 190-203, doi:https://doi.org/10.1016/j.ocemod.2015.10.009 (2016).

22 Lorenzo, M., I., V. M., Evangelos, V., Alessandro, D. \& Luc, F. Global changes of extreme coastal wave energy fluxes triggered by intensified teleconnection patterns. Geophysical Research Letters 44, 2416-2426, doi:doi:10.1002/2016GL072488 (2017).

23 Erikson, L. H., Hegermiller, C. A., Barnard, P. L., Ruggiero, P. \& van Ormondt, M. Projected wave conditions in the Eastern North Pacific under the influence of two CMIP5 climate scenarios. Ocean Modelling 96, 171-185, doi:https://doi.org/10.1016/j.ocemod.2015.07.004 (2015).

24 Bricheno, L. M. \& Wolf, J. Future wave conditions of Europe, in response to high-end climate change scenarios. Journal of Geophysical Research: Oceans $\mathbf{0}$, doi:doi:10.1029/2018JC013866 (2018).

25 Casas-Prat, M., Wang, X. L. \& Swart, N. CMIP5-based global wave climate projections including the entire Arctic Ocean. Ocean Modelling 123, 66-85, doi:https://doi.org/10.1016/j.ocemod.2017.12.003 (2018).

26 Semedo, A. et al. CMIP5-Derived Single-Forcing, Single-Model, and Single-Scenario WindWave Climate Ensemble: Configuration and Performance Evaluation. Journal of Marine Science and Engineering 6, 90 (2018).

27 Timmermans, B., Stone, D., Wehner, M. \& Krishnan, H. Impact of tropical cyclones on modeled extreme wind-wave climate. Geophysical Research Letters 44, 1393-1401, doi:10.1002/2016GL071681 (2017).

28 Shimura, T., Mori, N. \& Hemer, M. Variability and future decreases in winter wave heights in the Western North Pacific. Geophysical Research Letters 43, 2716-2722, doi:doi:10.1002/2016GL067924 (2016).

29 Wang, X. L., Feng, Y. \& Swail, V. R. Climate change signal and uncertainty in CMIP5-based projections of global ocean surface wave heights. Journal of Geophysical Research: Oceans 120, 3859-3871, doi:doi:10.1002/2015JC010699 (2015).

30 Camus, P. et al. Statistical wave climate projections for coastal impact assessments. Earth's Future 5, 918-933, doi:doi:10.1002/2017EF000609 (2017).

31 Hallegatte, S., Green, C., Nicholls, R. J. \& Corfee-Morlot, J. Future flood losses in major coastal cities. Nature Climate Change 3, 802, doi:10.1038/nclimate1979 https://www.nature.com/articles/nclimate1979\#supplementary-information (2013). Hemer, M., Wang, X., Webb, A. \& contributors, C. Report of the 2018 Meeting for the WCRP-JCOMM Coordinated Ocean Wave Climate Project (COWCLIP). (Paris, France, 2018). 
33 Hemer, M., Wang, X, Charles, E., Hegermiller, C., and COWCLIP contributors. Report of the 2014 Meeting for the WCRP-JCOMM Coordinated Global Wave Climate Projections (COWCLIP). (Paris, 2014).

34 Bricheno, L. \& Wolf, J. in Geophysical Research Abstracts Vol. 19 (2017).

35 Dee, D. P. et al. The ERA-Interim reanalysis: configuration and performance of the data assimilation system. Quarterly Journal of the Royal Meteorological Society 137, 553-597, doi:10.1002/qj.828 (2011).

36 Kar-Man Chang, E. CMIP5 Projected Change in Northern Hemisphere Winter Cyclones with Associated Extreme Winds. Journal of Climate 31, 6527-6542, doi:10.1175/JCLI-D-170899.1 (2018).

37 Karnauskas, K. B., Lundquist, J. K. \& Zhang, L. Southward shift of the global wind energy resource under high carbon dioxide emissions. Nature Geoscience 11, 38-43, doi:10.1038/s41561-017-0029-9 (2018).

38 Sigmond, M., Reader, M. C., Fyfe, J. C. \& Gillett, N. P. Drivers of past and future Southern Ocean change: Stratospheric ozone versus greenhouse gas impacts. Geophysical Research Letters 38, doi:doi:10.1029/2011GL047120 (2011).

39 Millar, R. J. et al. Emission budgets and pathways consistent with limiting warming to $1.5^{\circ} \mathrm{C}$. Nature Geoscience 10, 741, doi:10.1038/ngeo3031 https://www.nature.com/articles/ngeo3031\#supplementary-information (2017).

40 Sallenger Jr, A. H., Doran, K. S. \& Howd, P. A. Hotspot of accelerated sea-level rise on the Atlantic coast of North America. Nature Climate Change 2, 884, doi:10.1038/nclimate1597 https://www.nature.com/articles/nclimate1597\#supplementary-information (2012).

41 Sierra, J. P. \& Casas-Prat, M. Analysis of potential impacts on coastal areas due to changes in wave conditions. Climatic Change 124, 861-876, doi:10.1007/s10584-014-1120-5 (2014).

42 Shope, J. B., Storlazzi, C. D., Erikson, L. H. \& Hegermiller, C. A. Changes to extreme wave climates of islands within the Western Tropical Pacific throughout the 21 st century under RCP 4.5 and RCP 8.5, with implications for island vulnerability and sustainability. Global and Planetary Change 141, 25-38, doi:https://doi.org/10.1016/j.gloplacha.2016.03.009 (2016).

43 Wang, X. L. \& Swail, V. R. Climate change signal and uncertainty in projections of ocean wave heights. Climate Dynamics 26, 109-126, doi:10.1007/s00382-005-0080-x (2006).

44 Jacob, D. et al. EURO-CORDEX: new high-resolution climate change projections for European impact research. Regional Environmental Change 14, 563-578, doi:10.1007/s10113-013-0499-2 (2014).

45 Morim, J., Hemer, M., Andutta, F., Cartwrighta, N. \& Shimura, T. CMIP5 climate model surface winds: insights and footprint of model components. Submitted (2018).

46 Greve, P. et al. Global assessment of water challenges under uncertainty in water scarcity projections. Nature Sustainability 1, 486-494, doi:10.1038/s41893-018-0134-9 (2018).

47 Stopa, J. E. Wind forcing calibration and wave hindcast comparison using multiple reanalysis and merged satellite wind datasets. Ocean Modelling 127, 55-69, doi:https://doi.org/10.1016/j.ocemod.2018.04.008 (2018).

48 Stammer, D., Roderik van der Wal, Robert J. Nicholls \& Schlosser, P. WCRP/IOC Sea Level 2017 Conference Statement. International WCRP/IOC Conference 2017 - Regional Sea Level Changes and Coastal Impacts. (Columbia University, New York, US, 2017).

49 Ward, J. H. Hierarchical Grouping to Optimize an Objective Function. Journal of the American Statistical Association 58, 236-244, doi:10.1080/01621459.1963.10500845 (1963).

50 Wang, X. L., Feng, Y. \& Swail, V. R. Changes in global ocean wave heights as projected using multimodel CMIP5 simulations. Geophysical Research Letters 41, 1026-1034, doi:doi:10.1002/2013GL058650 (2014).

51 Storch, H. v. \& Zwiers, F. W. Statistical Analysis in Climate Research. (Cambridge University Press, 1999).

52 Bosshard, T. et al. Quantifying uncertainty sources in an ensemble of hydrological climateimpact projections. Water Resources Research 49, 1523-1536, doi:doi:10.1029/2011WR011533 (2013). 
53 Garcia, R. A., Burgess, N. D., Cabeza, M., Rahbek, C. \& Araújo, M. B. Exploring consensus in 21st century projections of climatically suitable areas for African vertebrates. Global Change Biology 18, 1253-1269, doi:doi:10.1111/j.1365-2486.2011.02605.x (2012).

54 Wessel, P. \& Smith, W. H. F. A global, self-consistent, hierarchical, high-resolution shoreline database. Journal of Geophysical Research: Solid Earth 101, 8741-8743, doi:10.1029/96JB00104 (1996).

55 Wolff, C. et al. A Mediterranean coastal database for assessing the impacts of sea-level rise and associated hazards. Scientific Data 5, 180044, doi:10.1038/sdata.2018.44 (2018). 